\title{
Psychiatric comorbidities in patients with major depressive disorder
}

\author{
This article was published in the following Dove Press journal: \\ Neuropsychiatric Disease and Treatment \\ 10 November 2014 \\ Number of times this article has been viewed
}

Papan Thaipisuttikul

Pichai Ittasakul

Punjaporn Waleeprakhon

Pattarabhorn Wisajun

Sudawan Jullagate

Department of Psychiatry, Faculty of Medicine, Ramathibodi Hospital, Mahidol University, Bangkok, Thailand
Background: Psychiatric comorbidities are common in major depressive disorder (MDD). They may worsen outcome and cause economic burden. The primary objective was to examine the prevalence of psychiatric comorbidities in MDD. The secondary objectives were to compare the presence of comorbidities between currently active and past MDD, and between patients with and without suicidal risk.

Methods: This was a cross-sectional study. A total of 250 patients with lifetime MDD and age $\geq 18$ years were enrolled. The Mini International Neuropsychiatric Interview (MINI), Thai version, was used to confirm MDD diagnosis and classify comorbidities. MDD diagnosis was confirmed in 190, and 60 patients were excluded due to diagnosis of bipolar disorder.

Results: Of the 190 MDD patients, $25.8 \%$ had current MDD and $74.2 \%$ had past MDD. Eighty percent were women. The mean age at enrollment was 50 years, and at MDD onset was 41 years. Most patients were married (53.2\%), employed (54.8\%), and had $\geq 12$ years of education $(66.9 \%)$. There were 67 patients $(35.3 \%)$ with one or more psychiatric comorbidities. Comorbidities included dysthymia (19.5\%), any anxiety disorders (21.1\%) (panic disorder [6.8\%], agoraphobia [5.8\%], social phobia [3.7\%], obsessive-compulsive disorder [OCD] [4.7\%], generalized anxiety disorder [5.3\%], and post-traumatic stress disorder [4.2\%]), alcohol dependence ( $0.5 \%)$, psychotic disorder (1.6\%), antisocial personality (1.1\%), and eating disorders $(0 \%)$. Compared with past MDD, the current MDD group had significantly higher OCD $(P<0.001)$, psychotic disorder $(P=0.048)$, past panic disorder $(P=0.017)$, and suicidal risk $(P<0.001)$. Suicidal risk was found in $32.1 \%$ of patients. Patients with suicidal risk had more comorbid anxiety disorder of any type $(P=0.019)$ and psychotic disorder $(P=0.032)$.

Conclusion: Several comorbidities were associated with MDD. Patients with active MDD had higher comorbid OCD, psychotic disorder, past panic disorder, and suicidal risk. Patients with suicide risk had higher comorbid anxiety and psychotic disorders.

Keyword: suicidal risk, active major depressive disorder, anxiety disorder, psychotic disorder

\section{Introduction}

Major depressive disorder (MDD) is common in Thailand. It can cause unnecessary suffering, impaired functioning, increased mortality, and excessive use of health care resources. ${ }^{1}$ From the 2008 Thailand National Survey, the lifetime prevalence of MDD was $2.7 \%$. Women had 1.5-fold greater prevalence than men. ${ }^{2}$ Psychiatric comorbidities are commonly associated with MDD. They may affect clinical course, ${ }^{3}$ treatment, ${ }^{4}$ and suicidal risk, and cause economic burden. ${ }^{5}$ Untreated comorbidities can increase both direct and indirect costs of MDD care. ${ }^{6,7}$ Therefore, detection and treatment of psychiatric comorbidities are crucial in management of MDD.

The most prevalent comorbidities are anxiety disorders, ${ }^{8}$ substance use disorders ${ }^{9}$ and other depressive disorders. Epidemiologic studies have shown that the prevalence
Correspondence: Pichai Ittasakul 270 Rama VI Road, Ratchathewi, Bangkok 10400, Thailand

Tel $+662201 \quad 1478$

Fax +66 22003277

Email pichai.itt@mahidol.edu 
of at least one lifetime anxiety disorder was $59 \%$ in patients with lifetime MDD, ${ }^{10}$ while the prevalence of substance use disorders in MDD was 14\%. ${ }^{9}$ The presence of comorbid MDD and anxiety disorders resulted in greater disease severity and diminished treatment response. ${ }^{11-13}$ Similarly, comorbid MDD and substance use disorders were associated with increased frequency and severity of depressive episodes and higher rates of suicide attempts. ${ }^{14}$ Other depressive disorders, such as dysthymia, can be found in up to $50 \%$ of patients with MDD. ${ }^{15}$ One study found that comorbid psychiatric disorders, especially panic, generalized anxiety disorder (GAD), substance use, and dysthymic disorders, accelerate treatment seeking for MDD. ${ }^{16}$

The clinical course of MDD can be affected by many factors. One study showed that severity of depression and level of comorbidities can affect the duration of depressive episodes and rate of recurrences. ${ }^{17}$ It seems psychiatric comorbidities can alter clinical manifestations of MDD, but how comorbidities run their clinical courses in relationship to MDD is still unknown. Most research in this area have been descriptive cross-sectional and have only focused on the presence of comorbidities in patients with lifetime MDD, regardless of their temporal relationship. This, in part, may be due to the assumption that both conditions are usually present at the same time. However, repeated studies found comorbid GAD and MDD to have different onsets and clinical courses. ${ }^{18,19}$

Suicidal behavior is an important issue in patients with MDD and anxiety disorders. Generally, patients with delusional depression are at high risk for suicide. ${ }^{20}$ One study in nonpsychotic MDD showed that concurrent social phobia and bulimia may be potential risk factors for suicide. ${ }^{21} \mathrm{~A}$ large longitudinal study to examine suicidal risk factors found comorbid anxiety disorders, personality disorders, and substance-related disorders to be associated with suicide attempts. ${ }^{22}$ A recent prospective study showed that patients with post-traumatic stress disorder (PTSD), MDD, intermittent depressive disorder, and epilepsy had shorter time to suicide attempt in univariate analysis. However, only depressive disorders were independent factors for suicidal risk in multivariate analysis. ${ }^{23}$ Increased risk for suicidal behavior was also related to alcohol abuse in patients with bipolar disorder. ${ }^{24}$ Epidemiologic study showed that depression and substance use disorders were risk factors for suicide for adults and adolescents in community. ${ }^{25}$

We hypothesized that different comorbidities may have different temporal relationships with a major depressive episode. The primary objective of this study was to examine the prevalence of psychiatric comorbidities in patients with lifetime MDD. The secondary objectives were to compare the presence of comorbidities between patients with currently active MDD and past MDD, and between patients with and without suicidal risk.

\section{Methods}

\section{Study design}

This was a cross-sectional study conducted in a tertiary care psychiatric outpatient clinic at Ramathibodi Hospital in Bangkok, Thailand, between October 2012 and January 2014. The protocol was approved by the Ethics Committee on Human Experimentation of the Institute. All subjects provided verbal and written informed consent prior to participation.

\section{Participants and procedure}

All patients seen in clinic for routine scheduled visits for MDD, consecutively, were asked to participate in the study. Eligible patients were $\geq 18$ years of age and had a lifetime diagnosis of MDD. Participants completed the questionnaire for baseline demographic data (age, sex, marital status, education, and employment status) and clinical characteristics (age of onset and duration of illness). Based on Diagnostic and Statistical Manual of Mental Disorders, Fourth Edition, Text Revision (DSM-IV-TR) criteria, MDD diagnosis was confirmed by two research assistants using Mini International Neuropsychiatric Interview (MINI) version 5, Thai version. Both assistants were trained to use the Thai version of MINI. Interrater reliability of scoring was assessed, and the agreement between raters was excellent $(\kappa=0.91) .{ }^{26}$ Participants with psychiatric or physical disorders that prevented them from being interviewed or undermined their ability to provide accurate information, and those who declined participation in the study were excluded.

\section{Measures}

Based on DSM-IV-TR criteria, MINI version 5, Thai version, was used to 1) confirm the diagnosis of current/past MDD, 2) exclude patients with manic episode (which indicates bipolar disorder), 3) classify psychiatric comorbidities, and 4) detect suicidal risk. Current MDD was defined as any MDD active major depressive episode within 2 weeks, whereas past MDD was defined as major depressive episode at any time prior to the past 2 weeks. Comorbidities were classified into the following based on their common existence with MDD: dysthymia, anxiety disorder, substance and alcohol use disorder, psychotic disorder, antisocial personality 
disorder, and eating disorder. Suicidal risk was assessed by the suicidality module of the MINI. The suicidality module is a six-item questionnaire that investigates the presence of prior suicidal attempts, suicidal ideation, and behavior in the past month. The total score is used to grade the suicidal risk (where score 1-5= low risk, score 6-9= moderate risk, and score $\geq 10=$ high risk). The MINI is a standardized clinical diagnostic interview schedule for DSM-IV Axis-I disorders. ${ }^{27}$ It can be reliably administered by trained interviewers. The Thai version was validated to use as a gold standard for diagnosis of comorbidities in psychiatric patients. ${ }^{28}$

\section{Statistical analysis}

We performed statistical analysis using SPSS 18.0 for Windows (IBM Corp., Armonk, NY, USA). Descriptive analysis was done to assess prevalence of psychiatric comorbidities in patients with MDD. Chi-square or Fisher's exact test was used to compare categorical data. Paired and independent $t$-tests or nonparametric tests were used to compare continuous data. We conducted univariate analysis to compare comorbidities between patients who currently had MDD and patients who had MDD in the past, and between patients with and without suicidal risk.

\section{Results}

\section{Sample description}

A total of 250 were enrolled. After initial assessment, 190 patients $(76.0 \%)$ had confirmed diagnosis of MDD by MINI, and 60 patients $(24.0 \%)$ were excluded due to the diagnosis of bipolar disorder. Of the 190 MDD patients, 141 (25.8\%) had current MDD and 49 (74.2\%) had past MDD. Eighty percent were women. Mean age at enrollment was 50 years (range 19-73 years) and at MDD onset was 41 years (range 12-67 years). Most patients were married (53.2\%), employed $(54.8 \%)$, and had 12 years of education or more $(66.9 \%)$ (Table 1$)$.

\section{Prevalence of psychiatric comorbidities}

There were 67 patients $(35.3 \%)$ who had one or more psychiatric comorbidity. Compared with patients without psychiatric comorbidity, those with comorbidity were younger (age 51.2 versus 47.4 years) $(t=2.0, d f=188.0$, $P=0.049$ ) and had earlier onset of MDD (age 42.6 versus 37.7 years $)(t=2.61, d f=187.0, P=0.01)$. There was no difference between the two groups on sex, marital status, education level, and employment status. Patients with psychiatric comorbidity had statistically higher suicidal risk

Table I Demographic data of patients with major depressive disorder

\begin{tabular}{|c|c|c|c|}
\hline \multirow{2}{*}{$\begin{array}{l}\text { Demographic and } \\
\text { clinical characteristics }\end{array}$} & \multicolumn{3}{|l|}{ Mean \pm SD or number (\%) } \\
\hline & No comorbidities $N=123$ & $\geq \mathbf{I}$ comorbidities $\mathbf{N}=67$ & Total $\mathbf{N}=190$ \\
\hline Age (years)* & $51.2 \pm 12.5$ & $47.4 \pm 13.1$ & $49.9 \pm 12.8$ \\
\hline \multicolumn{4}{|l|}{ Sex } \\
\hline Male & $26(21.1 \%)$ & $12(17.9 \%)$ & $38(20 \%)$ \\
\hline Female & 97 (78.9\%) & $55(82.1 \%)$ & 152 (80\%) \\
\hline \multicolumn{4}{|l|}{ Marital status } \\
\hline Single & 37 (30.1\%) & 21 (31.3\%) & $58(30.5 \%)$ \\
\hline Married or live together & $72(58.5 \%)$ & 35 (52.2\%) & 107 (56.3\%) \\
\hline Divorced/separated/death of spouse & 14 (1 I.4\%) & II (16.5\%) & 25 (I3.2\%) \\
\hline \multicolumn{4}{|l|}{ Education } \\
\hline Never attend school & I $(0.8 \%)$ & $2(3 \%)$ & $3(1.6 \%)$ \\
\hline Less than 12 years of education & $30(24.4 \%)$ & $15(22.4 \%)$ & $45(23.7 \%)$ \\
\hline 12 years of education or over & $92(74.8 \%)$ & $50(74.6 \%)$ & $142(74.7 \%)$ \\
\hline \multicolumn{4}{|l|}{ Employment status } \\
\hline Unemployed & 55 (44.7\%) & $18(26.9 \%)$ & $73(38.4 \%)$ \\
\hline Employed & $68(55.3 \%)$ & $49(73.1 \%)$ & $117(61.6 \%)$ \\
\hline Age of onset (years)** & $42.6 \pm 12.2$ & $37.7 \pm 12.9$ & $40.9 \pm 12.6$ \\
\hline Illness duration (years) & $8.6 \pm 6.9$ & $9.6 \pm 10.1$ & $9.0 \pm 8.1$ \\
\hline \multicolumn{4}{|l|}{ MDD } \\
\hline Current & $28(22.8 \%)$ & $21(31.3 \%)$ & 49 (25.8\%) \\
\hline Past & 95 (77.2\%) & $46(68.7 \%)$ & |4| (74.2\%) \\
\hline \multicolumn{4}{|l|}{ Suicidal risk within past month ${ }^{* * *}$} \\
\hline Yes & 31 (25.2\%) & $30(44.8 \%)$ & $6 \mathrm{I}(32.1 \%)$ \\
\hline No & $92(74.8 \%)$ & 37 (55.2\%) & $129(67.9 \%)$ \\
\hline
\end{tabular}

Notes: $* P=0.049, * * P=0.01$, and $* * * P=0.006$ were considered statistically significant. Abbreviations: SD, standard deviation; MDD, major depressive disorder. 
compared with patients without psychiatric comorbidity (44.8\% versus $25.2 \%)\left(\chi^{2}=7.6, d f=1.0, P=0.006\right)$ (Table 1$)$.

Comorbidities included dysthymia (19.5\%), any anxiety disorders $(21.1 \%)$, alcohol dependence $(0.5 \%)$, psychotic disorder (1.6\%), and antisocial personality (1.1\%). The anxiety disorder subgroups included panic disorder (6.8\%), agoraphobia (5.8\%), social phobia (3.7\%), obsessive-compulsive disorder (OCD) (4.7\%), GAD (5.3\%), and PTSD (4.2\%). Comorbid eating disorders were not found.

\section{Current MDD versus past MDD}

Compared with past MDD, patients with current MDD had significantly higher comorbid OCD $(P<0.001)$, psychotic dis$\operatorname{order}(P=0.016)$, and past history of panic disorder $(P=0.017)$. However, patients with past MDD had significantly higher comorbid dysthymia $(P<0.001)$ (Table 2$)$. Patients in current MDD group had higher suicidal risk $(P<0.001)(32.6 \%$ versus $15.6 \%$ were low risk, $8.2 \%$ versus $2.8 \%$ were moderate risk, and $16.3 \%$ versus $5 \%$ were high risk). There was no difference between the two groups in any demographic parameters shown in Table 1.

\section{Suicidal risk and psychiatric comorbidity}

There were 61 (32.1\%) patients who had suicidal risk within the past month. The number of patients classified as low, moderate, and high suicidal risk were 38 (20\%), 8 (4.2\%), and $15(7.9 \%)$, respectively. Since the number of patients in each group was low, all patients with suicidal risk were

Table 2 Psychiatric comorbidities and suicidal risk in patients with current MDD $(n=49)$ and past MDD $(n=|4|)$

\begin{tabular}{llll}
\hline Comorbidity & Current & Past & P-value \\
& MDD N (\%) & MDD N (\%) & \\
\hline Dysthymia & $\mathrm{I}(2 \%)$ & $36(25.5 \%)$ & $<0.00$ I* $^{\circ}$ \\
Current panic disorder & $0(0 \%)$ & $\mathrm{I}(0.7 \%)$ & 1.000 \\
Past panic disorder & $7(14.3 \%)$ & $6(4.3 \%)$ & $0.017^{*}$ \\
Agoraphobia & $5(10.2 \%)$ & $6(4.3 \%)$ & 0.125 \\
Social phobia & $4(8.2 \%)$ & $3(2.1 \%)$ & 0.053 \\
OCD & $7(14.3 \%)$ & $2(1.4 \%)$ & $<0.00 I^{*}$ \\
PTSD & $3(6.1 \%)$ & $5(3.5 \%)$ & 0.439 \\
Alcohol dependence & $1(2 \%)$ & $0(0 \%)$ & 1.000 \\
Psychotic disorders & $6(12.1 \%)$ & $6(4.3 \%)$ & $0.048 *$ \\
Generalized anxiety disorder & $\mathrm{I}(2 \%)$ & $9(6.4 \%)$ & 0.457 \\
Antisocial personality disorder & $\mathrm{I}(2 \%)$ & $\mathrm{I}(0.7 \%)$ & 0.450 \\
None & $28(57.1 \%)$ & $95(67.4 \%)$ & 0.197 \\
Suicidal risk & & & \\
$\quad$ Low & $16(32.6 \%)$ & $22(15.6 \%)$ & $<0.001 *$ \\
Medium & $4(8.2 \%)$ & $4(2.8 \%)$ & \\
High & $8(16.3 \%)$ & $7(5.0 \%)$ & \\
\hline
\end{tabular}

Note: $* P<0.05$ was considered statistically significant.

Abbreviations: MDD, major depressive disorder; OCD, obsessive-compulsive disorder; PTSD, post-traumatic stress disorder. analyzed together as "suicide risk positive" (Table 3). Patients with suicidal risk had more comorbid any anxiety disorders $(31.3 \%$ versus $16.3 \%)\left(\chi^{2}=5.5, d f=1.0, P=0.019\right)$ and psychotic disorder $(4.9 \%$ versus $0 \%)\left(\chi^{2}=6.4, d f=1.0\right.$, Fisher's exact test $P=0.032$ ).

\section{Discussion}

Our study showed that at least one-third of MDD patients had psychiatric comorbidity. The prevalence of psychiatric comorbidities in our study was consistent with previous work. ${ }^{8}$ We found high prevalence of comorbid dysthymia and anxiety disorders, especially panic disorder, agoraphobia, GAD, OCD, and PTSD. However, the prevalence of comorbid alcohol/substance use disorders was lower than previously reported. ${ }^{9}$ This can be explained by a higher proportion of women in our study as the prevalence of alcohol/ substance-related disorders is much higher in men. In addition, we had seen a very small number of substance-related patients in our psychiatric clinic, probably because of the availability and accessibility of special substance-related psychiatric facility nearby.

In order to get a cross-sectional picture of how psychiatric comorbidities are related to MDD, we compared the presence of comorbidities between current MDD and past MDD. Patients with current MDD had statistically higher comorbid OCD, psychotic disorder, and past history of panic disorder. OCD has been known to be a genetic and biological psychiatric disease, ${ }^{29,30}$ which is similar to MDD. Physicians should always look for comorbid OCD in patients with MDD and if present, not hesitate to treat. Generally, OCD has a more chronic course compared with MDD. One study found that patients with comorbid MDD has less remitting OCD. ${ }^{31}$ Another study found comorbid OCD with

Table 3 Suicidal risk and psychiatric comorbidity

\begin{tabular}{|c|c|c|c|}
\hline \multirow[t]{2}{*}{ Psychiatric comorbidity } & \multicolumn{2}{|c|}{ Suicidal risk, N (\%) } & \multirow[t]{2}{*}{$P$-value } \\
\hline & $\begin{array}{l}\text { Negative } \\
(\mathrm{N}=129)\end{array}$ & $\begin{array}{l}\text { Positive } \\
(\mathrm{N}=6 \mathrm{I})\end{array}$ & \\
\hline Dysthymia $(n=37)$ & $23(17.8 \%)$ & $14(23.0 \%)$ & 0.405 \\
\hline Any anxiety disorder $(n=40)$ & $21(16.3 \%)$ & 19 (31.3\%) & $0.019^{*}$ \\
\hline Panic disorder $(n=13)$ & $6(4.7 \%)$ & $7(11.5 \%)$ & 0.082 \\
\hline Agoraphobia $(n=I I)$ & $7(5.4 \%)$ & $4(6.6 \%)$ & 0.748 \\
\hline Social phobia $(n=7)$ & $3(2.3 \%)$ & $4(6.6 \%)$ & 0.214 \\
\hline Obsessive-compulsive disorder $(n=9)$ & $7(5.4 \%)$ & $2(3.3 \%)$ & 0.721 \\
\hline Generalized anxiety disorder $(n=10)$ & $4(3.1 \%)$ & $6(9.8 \%)$ & 0.078 \\
\hline Post-traumatic stress disorder $(n=8)$ & $3(2.3 \%)$ & $5(8.2 \%)$ & 0.114 \\
\hline Alcohol use disorder $(n=l)$ & $0(0 \%)$ & I (I.6\%) & 0.321 \\
\hline Psychotic disorder $(n=12)$ & $4(3.1 \%)$ & $8(13.1 \%)$ & $0.032 *$ \\
\hline Antisocial personality disorder $(n=2)$ & $\mathrm{I}(0.8 \%)$ & $\mathrm{I}(\mathrm{I} .6 \%)$ & 0.54 \\
\hline
\end{tabular}

Note: $* P<0.05$ was considered statistically significant. 
MDD had more severe symptoms and higher suicidal risk. ${ }^{32}$ Further longitudinal studies are needed to better explain the relationship between MDD and OCD.

Although the term "psychotic disorder" used in MINI is not specific, questions in the interview process were compatible with schizophrenia criteria. One possibility is that these patients may have had comorbid MDD and schizophrenia. Our finding was consistent with previous studies that found high prevalence of depression in patients with schizophrenia, especially in the peak of active psychotic phase. $^{33-35}$ Alternatively, these patients may fall into the "schizoaffective disorder" category. It was found that the majority of patients with comorbid schizophrenia and MDD had postpsychotic depression, in which depression was a reaction to psychosis. ${ }^{36}$ Traditional belief that comorbid depression in schizophrenia patients is associated with better outcome has been opposed by the newer studies in terms of chronicity and relapses. ${ }^{37,38}$

The reason for higher prevalence of past panic disorder in current MDD in our study is unclear; one possible explanation could be that the secondary depression developed after panic disorder in some patients. Furthermore, we found that patients with past MDD had higher comorbid dysthymic disorder than current MDD. The difference between the two groups probably reflects the difficulty in detecting dysthymia during active, full-blown MDD episodes.

Suicidal risk is one of the major concerns in MDD. It was present in approximately one-third of MDD in our study, although most patients were classified as in the low-risk group. Unsurprisingly, patients with current MDD definitely had higher suicide risk than did the past MDD group. We found that patients with any comorbid anxiety disorders and comorbid psychotic disorder had higher suicidal risk. This finding conforms to previous studies. ${ }^{22,39,40}$

\section{Limitations}

Our study has some noteworthy limitations. First, our sample size was small. Our patients were predominantly women and had later onset of MDD compared with most cohorts. Second, our study populations may not represent patients treated in the community, due to the tertiary care setting of our clinic. Third, our study can provide only a cross-sectional picture of comorbidities in patients with current or past MDD - how comorbidities run their courses is probably much more complex. Prospective study should provide more accurate and detailed relationship between them. Fourth, assessing suicidal risk as low, moderate, and high risk may have limited implication in clinical practice. Future study of suicidal variables, including suicidal ideation, suicidal behavior, and impulsivity may provide better useful information for clinicians. Fifth, assessing psychotic disorder by MINI was limited because it cannot diagnose specific psychotic disorders. Finally, many patients in the past MDD group had comorbid dysthymia which, in theory, may cause some difficulty when one tries to distinguish them from active MDD patients. On the other hand, it is even more difficult to identify comorbid dysthymia in patients with active MDD, as stated in the discussion above.

\section{Conclusion}

Psychiatric comorbidities were common in our study, especially anxiety and other mood disorders, for example, dysthymia, past history of panic disorder, agoraphobia, GAD, and OCD.

MDD patients with comorbidity were relatively younger, with earlier MDD onset, and had higher suicide risk compared with patients with no comorbidity. MDD patients with high suicide risk had more comorbid any anxiety disorders and psychotic disorders. Compared with past MDD, current MDD had higher comorbid OCD, psychotic disorder, and past history of panic disorder but less dysthymia.

\section{Acknowledgments}

Special thanks to Dr Rattana Saipanish for statistics recommendations and Dr Iyavut Thaipisuttikul for language check and manuscript preparation.

This study was supported by grants from the Faculty of Medicine, Ramathibodi Hospital, Mahidol University, Bangkok, Thailand.

\section{Disclosure}

The authors report no conflicts of interest in this work.

\section{References}

1. Bundhamcharoen K, Odton P, Phulkerd S, Tangcharoensathien V. Burden of disease in Thailand: changes in health gap between 1999 and 2004. BMC Public Health. 2011;11:53.

2. Kongsuk T. [The Prevalence of Major Depressive Disorders in Thailand: Results from the Epidemiology of Mental Disorders National Survey 2008]. Nonthaburi: Department of Mental Health; 2008. Available from: http://www.dmh.go.th/downloadportal/Morbidity/Depress2551. pdf. Accessed Sept 20, 2014. Thai.

3. Garcia-Toro M, Rubio JM, Gili M, et al. Persistence of chronic major depression: a national prospective study. J Affect Disord. 2013;151(1):306-312.

4. Riper H, Andersson G, Hunter SB, de Wit J, Berking M, Cuijpers P. Treatment of comorbid alcohol use disorders and depression with cognitivebehavioural therapy and motivational interviewing: a meta-analysis. Addiction. 2014;109(3):394-406.

5. McIntyre RS, Rosenbluth M, Ramasubbu R, et al; Canadian Network for Mood and Anxiety Treatments (CANMAT) Task Force. Managing medical and psychiatric comorbidity in individuals with major depressive disorder and bipolar disorder. Ann Clin Psychiatry. 2012;24(2):163-169. 
6. Asche CV, Joish VN, Camacho F, Drake CL. The direct costs of untreated comorbid insomnia in a managed care population with major depressive disorder. Curr Med Res Opin. 2010;26(8):1843-1853.

7. Schofield DJ, Callander EJ, Shrestha RN, Passey ME, Percival R, Kelly SJ. The indirect economic impacts of co-morbidities on people with depression. J Psychiatr Res. 2013;47(6):796-801.

8. Merikangas KR, Zhang H, Avenevoli S, Acharyya S, Neuenschwander M, Angst J; Zurich Cohort Study. Longitudinal trajectories of depression and anxiety in a prospective community study: the Zurich Cohort Study. Arch Gen Psychiatry. 2003;60(10):993-1000.

9. Gao K, Wang Z, Chen J, et al. Should an assessment of Axis I comorbidity be included in the initial diagnostic assessment of mood disorders? Role of QIDS-16-SR total score in predicting number of Axis I comorbidity. J Affect Disord. 2013;148(2-3):256-264.

10. Kessler RC, Merikangas KR, Wang PS. Prevalence, comorbidity, and service utilization for mood disorders in the United States at the beginning of the twenty-first century. Annu Rev Clin Psychol. 2007;3:137-158.

11. Penninx BW, Nolen WA, Lamers F, et al. Two-year course of depressive and anxiety disorders: results from the Netherlands Study of Depression and Anxiety (NESDA). J Affect Disord. 2011;133(1-2):76-85.

12. IsHak WW, Mirocha J, Christensen S, et al. Patient-reported outcomes of quality of life, functioning, and depressive symptom severity in major depressive disorder comorbid with panic disorder before and after ssri treatment in the star*d trial. Depress Anxiety. 2014;31(8):707-716.

13. Gili M, García Toro M, Armengol S, García-Campayo J, Castro A, Roca M. Functional impairment in patients with major depressive disorder and comorbid anxiety disorder. Can J Psychiatry. 2013;58(12):679-686.

14. Blanco C, Alegría AA, Liu SM, et al. Differences among major depressive disorder with and without co-occurring substance use disorders and substance-induced depressive disorder: results from the National Epidemiologic Survey on Alcohol and Related Conditions. J Clin Psychiatry. 2012;73(6):865-873.

15. King-Kallimanis B, Gum AM, Kohn R. Comorbidity of depressive and anxiety disorders for older Americans in the national comorbidity survey-replication. Am J Geriatr Psychiatry. 2009;17(9):782-792.

16. Olfson M, Liu SM, Grant BF, Blanco C. Influence of comorbid mental disorders on time to seeking treatment for major depressive disorder. Med Care. 2012;50(3):227-232.

17. Melartin TK, Rytsälä HJ, Leskelä US, Lestelä-Mielonen PS, Sokero TP, Isometsä ET. Severity and comorbidity predict episode duration and recurrence of DSM-IV major depressive disorder. J Clin Psychiatry. 2004;65(6):810-819.

18. Beesdo K, Pine DS, Lieb R, Wittchen HU. Incidence and risk patterns of anxiety and depressive disorders and categorization of generalized anxiety disorder. Arch Gen Psychiatry. 2010;67(1):47-57.

19. Lenze EJ, Mulsant BH, Mohlman J, et al. Generalized anxiety disorder in late life: lifetime course and comorbidity with major depressive disorder. Am J Geriatr Psychiatry. 2005;13(1):77-80.

20. Sadock BJ, Sadock VA. Kaplan and Sadock's Synopsis of Psychiatry: Behavioral Sciences/Clinical Psychiatry. 10th ed. Philadelphia, PA: Lippincott Williams \& Wilkins; 2007.

21. Morris DW, Trivedi MH, Husain MM, et al. Indicators of pretreatment suicidal ideation in adults with major depressive disorder. Acta Psychiatr Scand. 2010;121(6):480-484.

22. Bolton JM, Pagura J, Enns MW, Grant B, Sareen J. A population-based longitudinal study of risk factors for suicide attempts in major depressive disorder. J Psychiatr Res. 2010;44(13):817-826.

23. Uebelacker LA, Weisberg R, Millman M, Yen S, Keller M. Prospective study of risk factors for suicidal behavior in individuals with anxiety disorders. Psychol Med. 2013;43(7):1465-1474.
24. Oquendo MA, Currier D, Liu SM, Hasin DS, Grant BF, Blanco C. Increased risk for suicidal behavior in comorbid bipolar disorder and alcohol use disorders: results from the National Epidemiologic Survey on Alcohol and Related Conditions (NESARC). J Clin Psychiatry. 2010;71(7):902-909.

25. Wilcox HC. Epidemiological evidence on the link between drug use and suicidal behaviors among adolescents. Can Child Adolesc Psychiatr Rev. 2004;13(2):27-30.

26. Viera AJ, Garrett JM. Understanding interobserver agreement: the kappa statistic. Fam Med. 2005;37(5):360-363.

27. Sheehan DV, Lecrubier Y, Sheehan KH, et al. The Mini-International Neuropsychiatric Interview (M.I.N.I.): the development and validation of a structured diagnostic psychiatric interview for DSM-IV and ICD-10. J Clin Psychiatry. 1998;59(Suppl 20):S22-S33; quiz S34-S57.

28. Kittirathanapaiboon $\mathrm{P}$, Khamwongpin M. The validity of the Mini International Neuropsychiatric Interview (M.I.N.I.) Thai version. J Mental Health Thai. 2005;13(3):11.

29. Murphy DL, Moya PR, Fox MA, Rubenstein LM, Wendland JR, Timpano KR. Anxiety and affective disorder comorbidity related to serotonin and other neurotransmitter systems: obsessive-compulsive disorder as an example of overlapping clinical and genetic heterogeneity. Philos Trans R Soc Lond B Biol Sci. 2013;368(1615):20120435.

30. Hanna GL, Himle JA, Hanna BS, Gold KJ, Gillespie BW. Major depressive disorder in a family study of obsessive-compulsive disorder with pediatric probands. Depress Anxiety. 2011;28(6):501-508.

31. Marcks BA, Weisberg RB, Dyck I, Keller MB. Longitudinal course of obsessive-compulsive disorder in patients with anxiety disorders: a 15-year prospective follow-up study. Compr Psychiatry. 2011;52(6):670-677.

32. Viswanath B, Narayanaswamy JC, Rajkumar RP, et al. Impact of depressive and anxiety disorder comorbidity on the clinical expression of obsessive-compulsive disorder. Compr Psychiatry. 2012;53(6):775-782.

33. Buckley PF, Miller BJ, Lehrer DS, Castle DJ. Psychiatric comorbidities and schizophrenia. Schizophr Bull. 2009;35(2):383-402.

34. HäfnerH, Maurer K, Trendler G, anderHeiden W, Schmidt M, Könnecke R. Schizophrenia and depression: Challenging the paradigm of two separate diseases - A controlled study of schizophrenia, depression and healthy controls. Schizphr Res. 2005;77(1):11-24.

35. Tsai J, Rosenheck RA. Psychiatric comorbidity among adults with schizophrenia: a latent class analysis. Psychiatry Res. 2013;210(1):16-20.

36. Knights A, Hirsch SR. "Revealed" depression and drug treatment for schizophrenia. Arch Gen Psychiatry. 1981;38(7):806-811.

37. Sim K, Chan YH, Chong SA, Siris SG. A 24-month prospective outcome study of first-episode schizophrenia and schizoaffective disorder within an early psychosis intervention program. J Clin Psychiatry. 2007;68(9):1368-1376.

38. Tsuang D, Coryell W. An 8-year follow-up of patients with DSM-III-R psychotic depression, schizoaffective disorder, and schizophrenia. Am J Psychiatry. 1993;150(8):1182-1188.

39. Boden JM, Fergusson DM, Horwood LJ. Anxiety disorders and suicidal behaviours in adolescence and young adulthood: findings from a longitudinal study. Psychol Med. 2007;37(3):431-440.

40. Suttajit S, Paholpak S, Choovanicvong S, KittiwattanagulK, Pratoomsri W, Srisurapanont M. Correlates of current suicide risk among Thai patients with bipolar I disorder: findings from the Thai Bipolar Disorder Registry. Neuropsychiatr Dis Treat. 2013;9:1751-1757. 


\section{Publish your work in this journal}

Neuropsychiatric Disease and Treatment is an international, peerreviewed journal of clinical therapeutics and pharmacology focusing on concise rapid reporting of clinical or pre-clinical studies on a range of neuropsychiatric and neurological disorders. This journal is indexed on PubMed Central, the 'PsycINFO' database and CAS, and is the official journal of The International Neuropsychiatric Association (INA). The manuscript management system is completely online and includes a very quick and fair peer-review system, which is all easy to use. Visit http://www.dovepress.com/testimonials.php to read real quotes from published authors.

\footnotetext{
Submit your manuscript here: http://www.dovepress.com/neuropsychiatric-disease-and-treatment-journal
} 